\title{
Climatic niche shifts are common in introduced plants
}

\author{
Daniel Z. Atwater ${ }^{1,2 \star}$, Carissa Ervine ${ }^{2}$ and Jacob N. Barney ${ }^{2}$
}

\begin{abstract}
Our understanding of how climate influences species distributions and our ability to assess the risk of introduced species depend on the assumption that species' climatic niches remain stable across space and time. While niche shifts have been detected in individual invasive species, one assessment of $\sim 50$ plants in Europe and North America concluded that niche shifts were rare, while another concluded the opposite. These contradictory findings, limited in species number and geographic scope, leave open a need to understand how often introduced species experience niche shifts and whether niche shifts can be predicted. We found evidence of climatic niche shifts in $65-100 \%$ of 815 terrestrial plant species introduced across five continents, depending on how niche shifts were measured. Individual species responses were idiosyncratic, but we generally saw that niche shifts reflected changes in climate availability at the continent scale and were largest in long-lived and cultivated species. Smaller intercontinental niche shifts occurred within species' native ranges. Overall, the climatic niches of terrestrial plant species were not conserved as they crossed continents. These results have major consequences for applying environmental niche models to assess the risk of invasive species and for predicting species responses to climate change. Our findings challenge the tenet that species' niches are conserved aspects of their ecology.
\end{abstract}

\begin{abstract}
$t$ has long been assumed that a species' realized climatic niche, defined as the set of climates in which a species has a stable or growing population ${ }^{1,2}$, is an essential aspect of the ecology of that species. Thus, it has also been assumed that by modelling the climatic niche of a species, we should be able to predict its distribution following climate change or introduction to a new continent ${ }^{3,4}$. Accurate models are crucial for mitigating the damage caused by invasive species, assessing the invasion risks of introduced species and identifying threats to protected ecosystems $s^{5,6}$. However, either because of changes in their realized or fundamental climatic niche-defined as the set of climates in which a species can have a stable or growing population in the absence of limitation by biotic interactions, dispersal, or geography-introduced species may not occupy the same climatic niche in their native and introduced ranges (termed a 'niche shift'), complicating efforts to predict their future distributions.

Currently, we lack a clear understanding of the relative frequency and importance of climatic niche shifts in introduced species or whether niche shifts occur in the fundamental or realized niche ${ }^{7}$. We are even more limited in our ability to predict which species will experience niche shifts and in which directions shifts will occur. Climatic niche shifts have been observed in studies of individual species ${ }^{8-11}$ as well as in larger-scale studies of 26 invasive plants in Australia ${ }^{12}, 51$ invasive plants in North America ${ }^{13}$ and 128 reptiles and amphibians globally ${ }^{14}$. Conversely, other studies of 28 bird species ${ }^{15}$ and 50 plant species ${ }^{16}$ have reported that climatic niche shifts are rare. As the pace of species introductions increases, with severe impacts on global food production, human health, ecosystem services and biodiversity ${ }^{17,18}$, our inconsistent and incomplete understanding of the frequency of climatic niche shifts impairs our ability to forecast species dynamics $^{3}$. This has prompted urgent calls for globally comprehensive tests of niche conservatism and niche dynamics of larger numbers of introduced species ${ }^{3,19}$.
\end{abstract}

The niche dynamics of an introduced species include five processes: abandonment, pioneering, unfilling, expansion and stability (Fig. 1). Unfilling, expansion and stability concern 'analogue' niche space, which is defined as regions of climate space that are shared between the native and introduced range ${ }^{3}$, and may involve shifts in the fundamental and realized niche. Unfilling occurs when a species occupies a climate in its native range, but not in its introduced range. Unfilling may be caused by a change in the fundamental or realized niche, but is usually interpreted as evidence of dispersal limitation in the introduced range ${ }^{3,16}$. Thus, unfilling may be hypothesized to decline as invasions progress. Expansion occurs when a species occupies a climate in its introduced range, but not in its native range. If the native range is correctly identified (that is, as the area beyond which a species' dispersal is limited by geographic features, but within which it is not), expansion must result from either a change in the fundamental climatic niche or a change in biotic interactions (for example, release from native-range enemies or competitors ${ }^{20,21}$ ). Stability occurs in climates that are occupied in both the native and introduced ranges.

Niche dynamics may also occur in 'non-analogue' space, which is defined as climate space that is not shared between the native and introduced ranges ${ }^{3,13,22,23}$. For example, the introduced range may lack climates that were occupied in the native range-a process we refer to as niche abandonment (Fig. 1). Conversely, introduced species may pioneer novel climates in the introduced range that were not present in the native range. Abandonment and pioneering may not be considered shifts in the fundamental niche, as they do not reflect the action of ecological processes, but they may influence our ability to forecast species' responses to translocation and climate change. Niche pioneering could be of particular concern because it complicates our ability to extrapolate models into climates for which native-range data are unavailable.

Multiple methods are used to estimate climatic niche shifts ${ }^{3,24,25}$, and differences in the native- and introduced-range climatic

'Department of Biological Sciences, North Carolina State University, Raleigh, NC 27695, USA. ${ }^{2}$ Department of Plant Pathology, Physiology and Weed Science, Virginia Tech, Blacksburg, VA 24061, USA. *e-mail: danatwater@gmail.com 


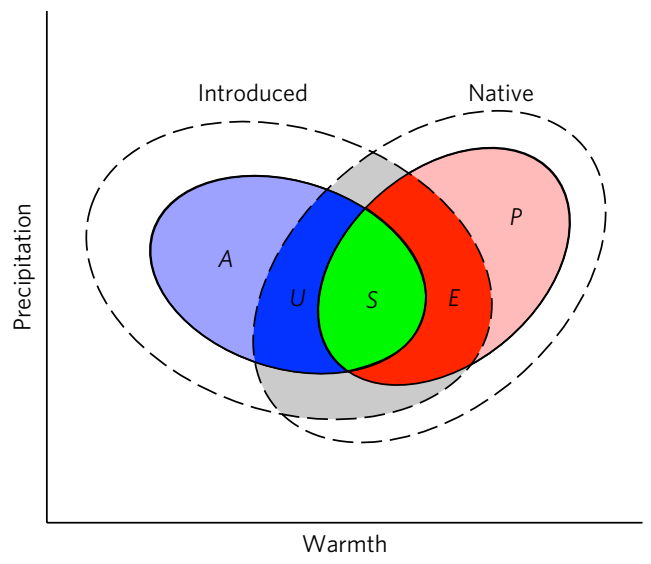

Fig. 1 | Native and introduced niche dynamics. Available climates (dashed lines) and occupied climates (solid lines) are shown for both a native and introduced range, with niche dynamics indicated by solid colours (light blue: abandonment $(A)$; dark blue: unfilling $(U)$; green: stability $(S)$; dark red: expansion $(E)$; and light red: pioneering $(P)$ ), with the analogue climate shown in grey. The axes show example climate variables in 'climate space', as opposed to geographic space. To analyse the magnitude of each of these dynamics, we sum the area of each dynamic, weighted by the availability of each climate. Modified from ref. ${ }^{3}$ with labels for abandonment and pioneering.

distributions are usually evaluated by estimating the climatic region encompassing a proportion of occurrences and evaluating the volume of climate space in each region ${ }^{13,15,16}$. In this study, we used ordination $^{25}$ (Supplementary Fig. 1) to evaluate niche dynamics in 815 terrestrial plant species (Supplementary Table 1). To do this, we plotted occurrences of each species in climate space separately in each of five biogeographic ranges (hereafter, 'continents': Australasia, Eurasia, North America, Sub-Saharan Africa and South America ${ }^{26}$ ). Next, we evaluated niche shifts between the global native (GN) range and each introduced-range (I) continent (GN-I comparisons; $n=1,561)$ and between each native-range $(\mathrm{N})$ and introducedrange continent ( $\mathrm{N}-\mathrm{I}$ comparisons; $n=2,364)$. For the 159 species with native ranges on multiple continents, we also evaluated niche shifts between native ranges on each continent ( $\mathrm{N}-\mathrm{N}$ comparisons; $n=244$ ). We explicitly considered both analogue and non-analogue climate space to evaluate the extent to which niche shifts were affected by changes in climate availability.

We tested niche shifts against three sets of null hypotheses: (1) we used resampling of both the native and introduced niche (SAMP) to determine whether observed niche shifts were likely to be real or caused by methodological biases and sampling processes, (2) we performed niche similarity (SIM) tests to determine whether a species' native and introduced niches were more similar to one another than to the niches of the other species in the database $e^{25,27}$ and (3) we performed niche equivalency (EQ) tests to determine whether native- and introduced-range models made equivalent predictions ${ }^{25,27}$. Each of these tests provided different information about the degree of niche overlap/stability between the native and introduced range. For example, a significant EQ test (where niche overlap was less than expected) meant that the native- and introduced-range climate models were not equivalent, yet a significant SIM test (where niche overlap was more than expected) showed that that species' native and introduced ranges, while different, were nonetheless similar compared with the niche variation among species. A significant SAMP test (where niche overlap was less than expected) told us whether the apparent niche shift was greater than that caused by our sampling and modelling procedures (which can introduce some bias; see Supplementary Fig. 2). We note that the
SAMP and SIM tests were variations on the 'niche similarity test' used in other studies ${ }^{25,27}$. Differences among null-hypothesis tests are described in greater detail below. In addition to these tests, we used linear models to investigate whether niche shifts varied according to species life span, growth form and cultivation status, and to compare $\mathrm{N}-\mathrm{I}$ and $\mathrm{N}-\mathrm{N}$ models.

We attempted to address a known limitation ${ }^{28}$ of the Global Biodiversity Information Facility (GBIF; http://www.gbif.org) and Early Detection and Distribution Mapping System (http://www. eddmaps.org) databases-poor coverage of Africa and Asiaby removing geographic sampling bias from our niche models (Supplementary Fig. 1). However, our method of removing sampling bias required the assumption that all species were subject to the same sampling bias and that bias was represented by the overall distribution of occurrences in the dataset. While such violations may have influenced our results, as global data quality improves, the accuracy of niche models and our estimates of niche dynamics will also improve.

Using these comparisons, we investigated (1) how often introduced species experienced niche shifts, (2) whether niche shifts resulted primarily from expansion or unfilling, (3) whether the niche was conserved throughout species' native ranges, (4) whether the direction of niche shifts was influenced by climate availability and (5) whether species growth form and life span influenced the magnitude of niche shifts. With a database of 815 species, we were able to comprehensively test climate niche dynamics and go beyond analogue climate comparisons to identify contributions of climate, geography and species life histories to climatic niche shifts.

\section{Results and discussion}

Commonness of niche shifts. We observed overwhelming evidence of niche shifts. Niche stability $(S)$, measured as the portion of the analogue climate volume occupied by a species in both the native and introduced range, accounted for only about $25 \%$ of the GN-I and N-I (Table 1) analogue niche dynamics and was lower than expected, based on native-range models, for $65-100 \%$ of 815 species and 38-97\% of biogeographic comparisons (SAMP and EQ tests; Table 1). Likewise, Schoener's $D$ (ref. ${ }^{29}$ ), a robust metric of niche overlap ${ }^{30}$ that falls between zero (no overlap) and one (perfect overlap), was significantly lower than expected for $43-99.7 \%$ of GN-I and N-I comparisons ( $D_{\text {Raw }}$; Table 1 and Fig. 2). Overlap was slightly higher in climate space weighted according to introducedrange climate commonness $\left(D_{\text {Clim }}\right)$ and geographic sampling effort $\left(D_{\mathrm{Geo}}\right)$, indicating that niche overlap was greater in more common and more commonly sampled climates. Nearly all of the EQ tests for overlap and stability were significant, meaning that native- and introduced-range models were almost never equivalent. The less sensitive SAMP tests indicated significantly lower niche stability than resampled data for $38 \%$ of N-I comparisons and $53 \%$ of GN-I comparisons. In only $14-18 \%$ of SIM comparisons was the observed stability or overlap greater than expected. Thus, most species native and introduced ranges were dissimilar enough that it appeared as though they belonged to different species.

Prevalence of unfilling and expansion. It has been suggested that niche shifts in introduced species result primarily from unfilling ${ }^{15,16}$, as it takes time for introduced species to colonize all suitable niche space $^{13,31,32}$. Such colonization lags could be caused by dispersal limitation, but they could also occur as colonizing populations slowly recover genetic diversity that was lost due to founder effects; for example, as populations evolve along latitudinal clines ${ }^{33}$. In the case of dispersal limitation, the fundamental niche remains unaltered and unfilling converts to stability as species overcome it. In the case of recovered genetic diversity, species experience an initial contraction of the fundamental niche followed by re-enlargement of the fundamental niche via evolution. If this niche enlargement 


\begin{tabular}{|c|c|c|c|c|c|c|c|}
\hline Comparison & Metric & Mean value & $n$ & SAMP & SIM & $n$ & EQ \\
\hline \multirow[t]{4}{*}{ GN-I } & $D_{\text {Raw }}$ & $0.314 \pm 0.185$ & 1,561 & $43.6 \%$ & $14.2 \%$ & 1,545 & $95.6 \%$ \\
\hline & $D_{\text {Clim }}$ & $0.336 \pm 0.202$ & 1,561 & $46.3 \%$ & $14.4 \%$ & 1,545 & $94.0 \%$ \\
\hline & $D_{\mathrm{Geo}}$ & $0.404 \pm 0.216$ & 1,561 & $61.2 \%$ & $15.6 \%$ & 1,545 & $91.3 \%$ \\
\hline & $S$ & $0.341 \pm 0.238$ & 1,553 & $37.7 \%$ & $14.8 \%$ & 1,545 & $90.6 \%$ \\
\hline \multirow[t]{4}{*}{$N-I$} & $D_{\text {Raw }}$ & $0.339 \pm 0.199$ & 2,364 & $42.6 \%$ & $18.4 \%$ & 1,965 & $99.7 \%$ \\
\hline & $D_{\text {Clim }}$ & $0.354 \pm 0.212$ & 2,364 & $45.6 \%$ & $17.5 \%$ & 1,965 & $99.1 \%$ \\
\hline & $D_{\mathrm{Geo}}$ & $0.407 \pm 0.228$ & 2,364 & $56.7 \%$ & $17.6 \%$ & 1,965 & $99.1 \%$ \\
\hline & $S$ & $0.313 \pm 0.269$ & 2,364 & $52.6 \%$ & $17.8 \%$ & 1,946 & $97.4 \%$ \\
\hline \multirow[t]{4}{*}{$\mathrm{N}-\mathrm{N}$} & $D_{\text {Raw }}$ & $0.400 \pm 0.189$ & 244 & $34.0 \%$ & $24.2 \%$ & 244 & $100.0 \%$ \\
\hline & $D_{\text {Clim }}$ & $0.413 \pm 0.197$ & 244 & $35.2 \%$ & $17.2 \%$ & 244 & $99.6 \%$ \\
\hline & $D_{\mathrm{Geo}}$ & $0.417 \pm 0.200$ & 244 & $44.3 \%$ & $18.4 \%$ & 244 & $98.4 \%$ \\
\hline & $S$ & $0.357 \pm 0.271$ & 244 & $25.5 \%$ & $22.5 \%$ & 239 & $96.7 \%$ \\
\hline
\end{tabular}

The mean \pm s.d. is provided for each variable. Results of full-null hypotheses (SAMP, $n=120$ ), niche similarity (SIM, $n=91-979)$ and niche equivalency (EQ, $n=200$ ) tests are shown. The number of evaluated comparisons is given. SAMP tests show the percentage of cases with less niche overlap than randomly resampled data. SIM tests compare niche overlap or stability within a species with niche overlap or stability between each species and each other species. A greater percentage means more cases had significant niche similarity (that is, a significant SIM test). The EQ tests show the percentage of cases in which the compared models cannot be considered equivalent. All are one-way tests evaluated at $\alpha=0.050$.

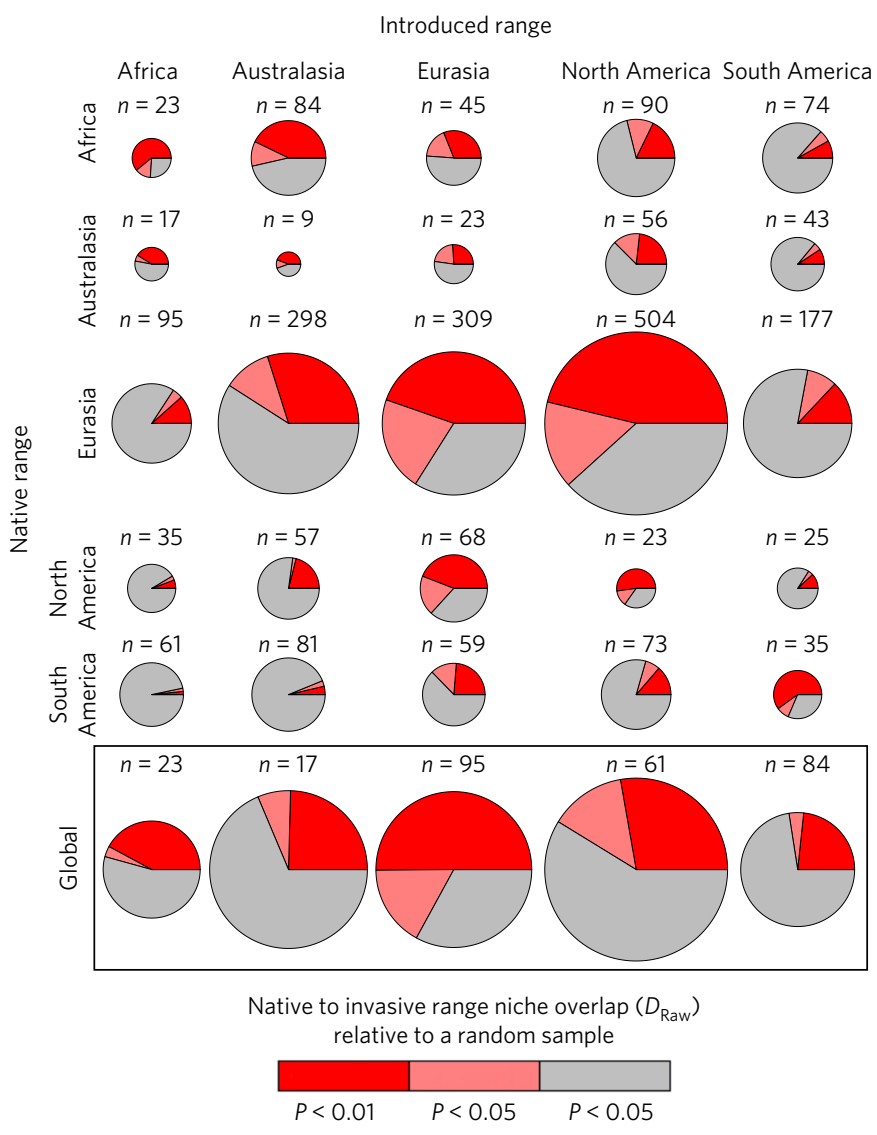

Fig. 2 | SAMP tests of niche overlap. Proportion of $\mathrm{N}-\mathrm{I}$ comparisons with significantly less niche overlap $(D)$ than expected compared with 120 random samples (SAMP tests; red: $P \leq 0.008$; pink: $P \leq 0.050$; one-way test). The area of the pie corresponds to the number of species in each comparison.

restores the original niche, it results in stability. However, if enlargement occurs in a novel direction, it results in niche expansion and pioneering ${ }^{34}$. Expansion can also occur as a result of different biotic interactions in the introduced range ${ }^{20,21}$ that alter the realized niche or erroneous estimation of the native range, which can cause pioneering to be mistaken for expansion. Unfilling $(U)$ and expansion $(E)$, measured here as the proportion of analogue climate volume in which either expansion or unfilling occurred, were common in both the GN-I and N-I comparisons (Table 2 and Fig. 3). While species varied, on average, $E$ and $U$ were similar in magnitude in the $\mathrm{N}-\mathrm{I}$ comparisons, while $E$ was greater than $U$ in the $\mathrm{GN}-\mathrm{I}$ comparisons.

Niche dynamics in non-analogue climate space were important. Abandonment (Fig. 1) accounted for approximately $23 \%$ of the overall N-I niche dynamics (Table 2 and Fig. 3; these were not assessed for the GN-I comparisons). This is ecologically interesting because it means that species leave large portions of their realized niche behind when they cross continents. For distribution modelling of invasive species, however, niche abandonment is not a concern because it does not affect our ability to forecast species distributions in a new range. The non-analogue niche dynamic much more relevant to invasion modelling is niche pioneering because of its potential to interfere with invasive-range niche forecasts. In this study, we found that niche pioneering was significant for only $9.8 \%$ of $\mathrm{N}-\mathrm{I}$ comparisons and was small on average (accounting for only $10 \%$ of the total niche dynamics). Thus, for many species, pioneering may not be likely to cause a problem in predictive modelling. However, for some species, pioneering was significant, potentially challenging predictive modelling efforts. Also, we hypothesize that pioneering could increase over time as colonization progresses.

Interestingly, unfilling was particularly pronounced for species introduced to Eurasia ( $U: 0.578 \pm 0.309)$ compared with other species ( $U: 0.310 \pm 0.276$ ), also leading to lower niche overlap and stability $\left(D_{\text {Raw }}: 0.185 \pm 0.141\right.$ versus $0.381 \pm 0.192 ; S: 0.116 \pm 0.131$ versus $0.366 \pm 0.272$ ). This was true for the $\mathrm{N}-\mathrm{I}$ (shown) and GN-I comparisons. The cause of low niche overlap in species introduced to Eurasia was not clear. Eurasian-introduced species were nearly perfectly representative of the larger dataset in growth form, life span and cultivation status and we did not have problems, in general, fitting models to Eurasian data. Thus, we conclude that this pattern was present in the source data.

It is important to note that our estimates of niche dynamics represented a snapshot in time because both the occurrence data and climate data used in this study span only a few decades. For example, human actions might have caused a reduction in the realized niche of a species. If that species was able to occupy more of its fundamental niche in the introduced range, we would detect that difference as niche expansion. Also, changing climatic conditions in 
Table 2 | Results of SAMP tests $(n=120)$ of various niche shift metrics

\begin{tabular}{|c|c|c|c|c|c|c|c|c|c|c|c|c|}
\hline \multirow[t]{2}{*}{ Metric } & \multicolumn{4}{|c|}{ GN-I } & \multicolumn{4}{|c|}{$\mathrm{N}-\mathrm{I}$} & \multicolumn{4}{|c|}{$\mathrm{N}-\mathrm{N}$} \\
\hline & Mean value & $n$ & Lower & Higher & Mean value & $n$ & Lower & Higher & Mean value & $n$ & Lower & Higher \\
\hline$A$ & - & - & - & - & $0.225 \pm 0.282$ & 2,364 & $30.3 \%$ & $21.7 \%$ & $0.288 \pm 0.287$ & 244 & $41.0 \%$ & $29.5 \%$ \\
\hline$U$ & $0.355 \pm 0.272$ & 1,553 & $21.5 \%$ & $21.9 \%$ & $0.367 \pm 0.304$ & 2,364 & $12.6 \%$ & $28.5 \%$ & $0.328 \pm 0.328$ & 244 & $13.1 \%$ & $33.2 \%$ \\
\hline$E$ & $0.304 \pm 0.261$ & 1,553 & $30.2 \%$ & $3.3 \%$ & $0.320 \pm 0.291$ & 2,364 & $11.1 \%$ & $29.4 \%$ & $0.315 \pm 0.277$ & 244 & $18.0 \%$ & $25.8 \%$ \\
\hline$P$ & - & - & - & - & $0.101 \pm 0.178$ & 2,364 & $42.7 \%$ & $9.8 \%$ & $0.298 \pm 0.279$ & 244 & $40.2 \%$ & $31.1 \%$ \\
\hline Centroid (RCA1) & $0.003 \pm 0.046$ & 1,553 & $31.7 \%$ & $5.0 \%$ & $0.000 \pm 0.033$ & 2,364 & $42.7 \%$ & $18.0 \%$ & $-0.002 \pm 0.025$ & 244 & $27.5 \%$ & $19.3 \%$ \\
\hline Centroid (RCA2) & $0.010 \pm 0.050$ & 1,553 & $26.7 \%$ & $10.9 \%$ & $0.005 \pm 0.039$ & 2,364 & $21.8 \%$ & $25.3 \%$ & $-0.001 \pm 0.032$ & 244 & $31.1 \%$ & $22.1 \%$ \\
\hline
\end{tabular}

This table shows the percentage of cases with significantly lower $(P \leq 0.025)$ and higher $(P \geq 0.975)$ values than randomly resampled data.

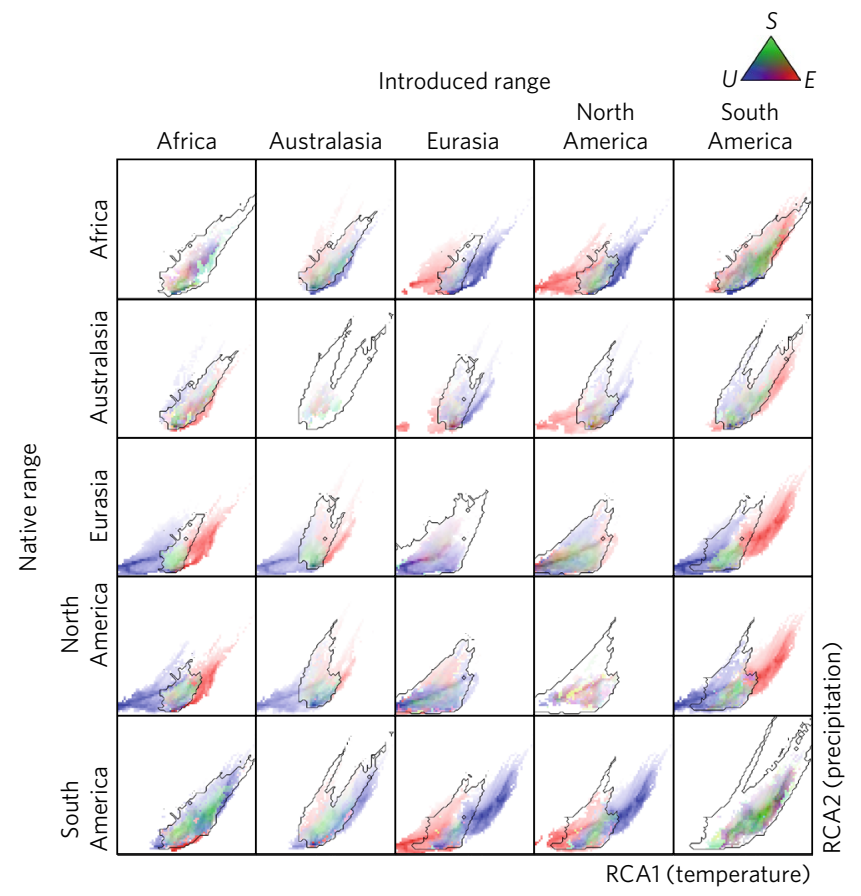

Fig. 3 | Niche dynamics of $\mathbf{N}$-I comparisons in climate space. The results are averaged among 815 species. The solid black lines outline the analogue climate space. Red inside the analogue space represents expansion $(E)$ and red outside the analogue space represents pioneering. Blue inside the analogue space represents unfilling $(U)$ and blue outside the analogue space represents abandonment. Green represents stability (S). Niche dynamics in less common climates fade to white.

the native and introduced ranges over centuries could create niche pioneering or abandonment.

The diversity of niche dynamics we observed suggests several potential causes, including dispersal limitation, evolutionary changes in the fundamental niche and/or altered biotic interactions in the introduced range. We hypothesize that niche shifts likely reflect a combination of these three processes, but experimentation is necessary to elucidate the processes that drive the niche dynamics of introduced species.

Native-range niche shifts. While the $\mathrm{N}-\mathrm{I}$ and GN-I comparisons enabled ustodetectnicheshiftsin recentlyintroducedspecies, the N-N comparisons allowed us to evaluate niche dynamics in species that experienced prehistoric, natural colonization of different continents. Niche overlap was higher for the $244 \mathrm{~N}-\mathrm{N}$ comparisons than for the N-I comparisons (Table 3), but $43 \%$ of $\mathrm{N}-\mathrm{N}$ comparisons also had lower niche overlap than would be expected due to chance in the SAMP tests, and all N-N comparisons had less overlap than expected in the EQ tests (Table 1). These data reveal that species' climatic niches can vary substantially among continents in their native range. The somewhat higher niche overlap in $\mathrm{N}-\mathrm{N}$ (which reflects prehistoric continental crossings) than $\mathrm{N}-\mathrm{I}$ and $\mathrm{GN}-\mathrm{I}$ comparisons (which reflect recent crossings) could be explained if intercontinental niche overlap increased over time, perhaps as species overcome dispersal barriers following introduction, or by the accumulation of genetic variance lost during colonization ${ }^{33}$. However, niche convergence was not complete, as evidenced by the SIM tests, which indicated high niche dissimilarity even in the $\mathrm{N}-\mathrm{N}$ comparisons (Table 1). This finding is important for two reasons. First, it means that the native ranges on different continents may not necessarily be treated as equivalent for the sake of distribution modelling. Second, it means that the climatic niche on one continent may not be inferred from the climatic niche on another continent, even if both continents are considered part of the native range. These findings paint a complex picture of a species' global niche as something that simultaneously must be considered to exist on the global scale, but also must be recognized to vary according to biogeographic factors at the continent scale.

As niche unfilling is hypothesized to convert to stability as colonizing populations expand, we hypothesized that there would be relatively less unfilling in the $\mathrm{N}-\mathrm{N}$ comparisons than in the $\mathrm{N}-\mathrm{I}$ comparisons. This was partially supported by our observations. Stability was significantly higher in the N-I comparisons than in the $\mathrm{N}-\mathrm{N}$ comparisons and, while unfilling was less in the $\mathrm{N}-\mathrm{N}$ than the N-I comparisons, as predicted, it nonetheless constituted a large portion of the $\mathrm{N}-\mathrm{N}$ niche dynamics. Also, the degree of expansion was similar between the $\mathrm{N}-\mathrm{N}$ and $\mathrm{N}-\mathrm{I}$ comparisons (Table 3), meaning niche expansion is likely to be common and perhaps persistent when species cross continents. This is a crucial finding because it gives us reason to believe that the realized niche in the introduced range may never fully converge on the nativerange niche.

Niche shift directionality. Niche shift directionality was estimated by measuring changes in the niche centroid (that is, the centre of mass of the climate niche) between continents. Centroid shifts were significant for most N-I comparisons (40-94\%) and GN-I comparisons (37-95\%) along both climate axes. Introduced species tended to move towards hotter, wetter climates, although this varied enormously among species (Table 2 ). When considering all $\mathrm{N}-\mathrm{I}$ comparisons together, centroid shifts in both temperature axes weakly but significantly reflected differences in climate availability (Fig. 4), supporting the hypothesis that, broadly speaking, niche shifts positively-albeit weakly-reflected changes in climate availability across continents. 
Table 3 | Comparisons between $\mathrm{N}-\mathrm{I}$ and $\mathrm{N}-\mathrm{N}$ models

\begin{tabular}{lllll} 
& N-I (mean \pm s.e. & N-N (mean \pm s.e.) & F & P \\
\hline$D_{\text {Raw }}$ & $0.338 \pm 0.018$ & $0.385 \pm 0.022$ & $13.41_{1,2472.3}$ & $<0.001$ \\
$D_{\text {Clim }}$ & $0.358 \pm 0.019$ & $0.404 \pm 0.023$ & $10.91_{1,250.9}$ & $<0.001$ \\
$D_{\text {Geo }}$ & $0.409 \pm 0.019$ & $0.401 \pm 0.023$ & $0.28_{1,2532.6}$ & 0.598 \\
$A$ (transformed) & $0.426 \pm 0.060$ & $0.350 \pm 0.062$ & $14.95_{1,251.0}$ & $<0.001$ \\
$U$ (transformed) & $0.633 \pm 0.042$ & $0.545 \pm 0.048$ & $12.57_{1,2578.8}$ & $<0.001$ \\
$S$ (transformed) & $0.527 \pm 0.037$ & $0.616 \pm 0.042$ & $16.80_{1,2503.3}$ & $<0.001$ \\
$E$ (transformed) & $0.564 \pm 0.025$ & $0.580 \pm 0.034$ & $0.38_{1,237.0}$ & 0.536 \\
$P$ (transformed) & $0.249 \pm 0.041$ & $0.424 \pm 0.044$ & $108.34_{1,257.4}$ & $<0.001$ \\
Centroid (RCA1) & $-0.002 \pm 0.002$ & $-0.004 \pm 0.003$ & $0.86_{1,237.6}$ & 0.355 \\
Centroid (RCA2) & $0.005 \pm 0.003$ & $-0.031 \pm 0.004$ & $9.31_{1,2307.3}$ & 0.002 \\
\hline
\end{tabular}

Least-squared means \pm s.e. are shown, from which random effects of species and biogeographic comparison have been removed. Some data were angular transformed to meet normality assumptions of the linear mixed models. Test statistics were generated using Kenward-Rogers approximation with linear mixed models. Degrees of freedom are shown as subscripts beside the $F$ statistics, with commas separating the numerator and denominator degrees of freedom.
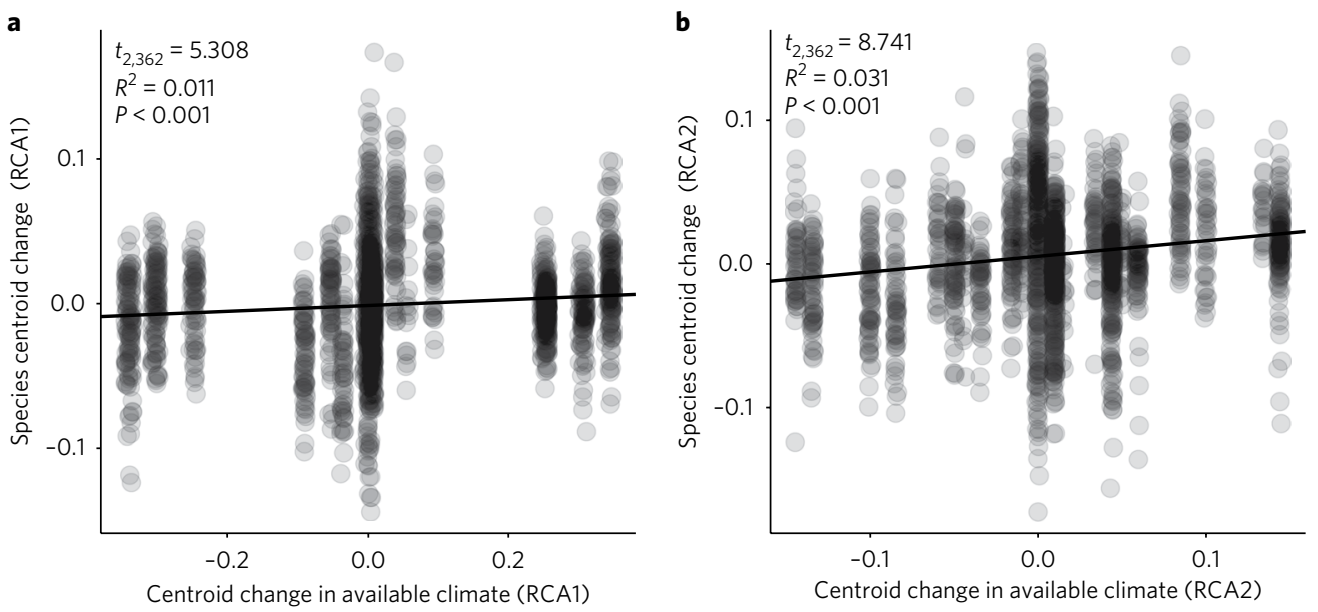

Fig. 4 | Climatic niche shifts reflect changes in climate availability. The centroid of the total available-climate space differs among continents. Changes in the centroid of a species' realized niche relate to changes in the available-climate centroid as it crosses continents. a,b, Centroid shifts in RCA1 (temperature and seasonality; $\mathbf{a}$ ) and RCA2 (precipitation; b) are shown, along with the results of linear models controlled for sampling effects using randomly resampled data. Points are jittered horizontally.

Effects of species traits on niche dynamics. The direction and magnitude of niche shifts depended on species growth form, life span and cultivation status (Table 4 ). In the N-I comparisons, herbaceous species showed greater niche overlap $\left(D_{\text {Raw }}\right)$ than woody plants, with woody species also showing lower stability and more expansion (Fig. 5). We further observed pronounced expansion in cultivated species (Fig. 5), which experienced lower niche overlap and less stability than uncultivated species. Species life history, growth form and cultivation status were strongly correlated, with a disproportionate number of cultivated species being long-lived and woody (Supplementary Table 2). Even with 815 species, we could not disentangle the independent effects of growth form, life span and cultivation status on niche dynamics. We hypothesize that niche shifts in long-lived species were influenced by cultivation, which allowed them to occupy otherwise unsuitable climates, partially supported by the fact that short-lived, non-cultivated species tended to shift towards wetter environments in their introduced range, whereas long-lived, cultivated species did not (Fig. 5). Our results indicate that human activities have the potential to dramatically modify niche dynamics in introduced species, particularly in cultivated species. They also reveal that species traits may have enormous and potentially predictable effects on their climatic niche dynamics.

\section{Summary}

The question of whether and how often introduced species' climatic niches are conserved has emerged as a major debate in ecology and conservation $^{3,16}$, with direct relevance to our ability to predict the future distribution of introduced species ${ }^{3,4,35}$, our ability to assess the invasion risk of introduced species ${ }^{5,6}$ and our fundamental understanding of how biogeography influences the evolution and ecology of species ${ }^{19}$. To date, tests of niche conservatism have been limited in scope, with the few larger-scale comparisons-involving 26-128 species-producing conflicting results ${ }^{12-16}$. In this study, we saw evidence of broad, general climatic niche shifts and we found that native- and introduced-range niche models were rarely, if ever, equivalent and were often so dissimilar that they appeared to belong to different species. Our findings that shifts in the realized niche were both overwhelmingly common and sometimes large and that species had the ability to utilize climatic niches that were not present in their home range have critical implications for our ability to predict species' introduced distributions based on native-range data $^{3,4,35}$. Encouragingly, we observed consistent trends in the direction and magnitude of niche shifts that depended on species growth forms and life history traits, suggesting that niche dynamics may be predictable in models that account for species traits. We propose that shifts in the climatic niche of species are a common and 
Table 4 | Tests of the effects of species life span, growth form and cultivation status on niche dynamics

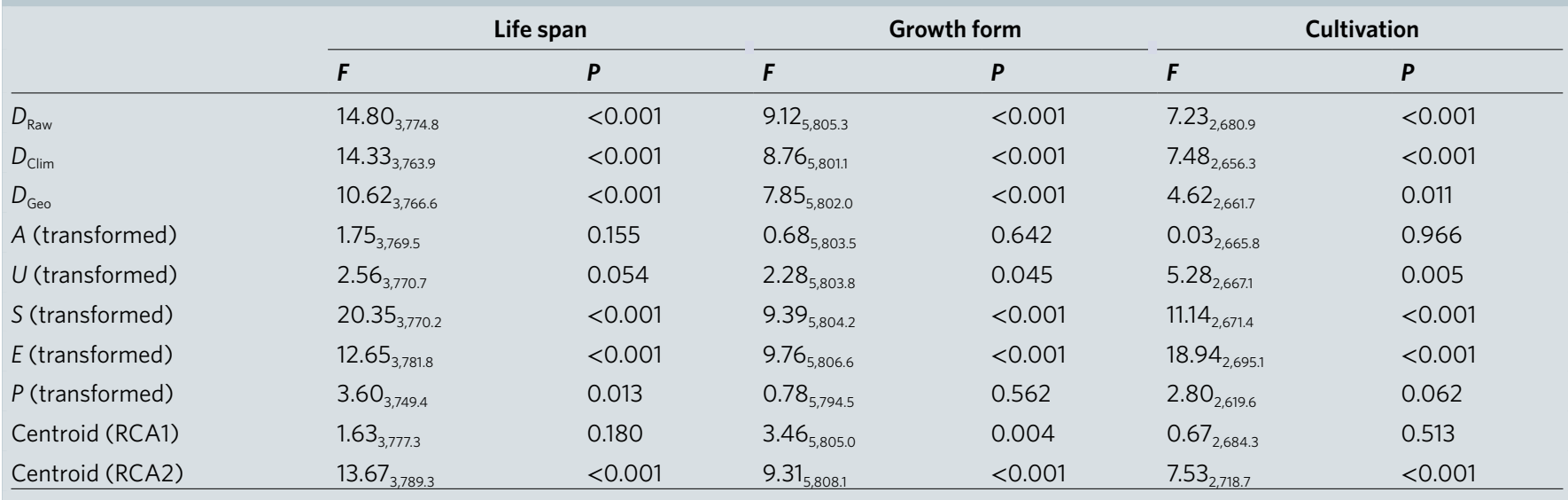

Some data were arcsine-square-root transformed to meet normality assumptions of the linear mixed models. Test statistics were generated using Kenward-Rogers approximation with linear mixed models. Degrees of freedom are shown as subscripts beside the $F$ statistics, with commas separating the numerator and denominator degrees of freedom.

expected consequence of intercontinental range expansion, resulting from a complex set of processes, including dispersal limitation and changes in species' fundamental climatic niche and biotic environment, and that we can predict species' future distributions based on species' traits and differences in climates between continents. Lastly, cross-continent niche shifts in native species suggest that niche shifts may be persistent, meaning that successful predictions of the future range of introduced species must anticipate niche shifts and not assume that they will lessen over time.

\section{Methods}

General approach. In this study, we used ordination to model changes in the climatic niches of introduced species (summarized in Supplementary Fig. 1). To do this, we calculated frequency distributions describing the expected probability of a species occurring in each combination of two climate component variables (temperature and precipitation) in both its native and introduced ranges. Importantly, we adjusted these frequency distributions to account for both climate and geographic sampling biases caused by unequal representation of different climates on different continents and by geographic variation in the sampling effort. We then compared the native and introduced frequency distributions. We compared the global native range with the introduced range on every continent (GN-I comparisons) and the native range on every continent with the introduced range on every continent $(\mathrm{N}-\mathrm{I})$. We also compared the native range of a species on one continent with its native range on another continent if that species was native to multiple continents $(\mathrm{N}-\mathrm{N})$. Comparisons involved metrics of niche overlap $(D)$, stability $(S)$, expansion $(E)$, unfilling $(U)$, pioneering $(P)$ and abandonment $(A)$.

Hypothesis testing. We employed three types of null-hypothesis test to determine whether niche shifts were statistically significant: SAMP, SIM ${ }^{25,27}$ and $\mathrm{EQ}^{25,27}$ A significant SAMP test meant that the observed niche dynamic did not result purely from sampling artefacts and methodological biases (which may be significant; see Supplementary Fig. 2). The null hypothesis was generated by measuring niche overlap between bias-corrected niches randomly resampled from the total set of all species occurrences in the native $\left(\mathrm{N}_{\text {Samp }}\right)$ and introduced ranges $\left(\mathrm{I}_{\mathrm{Samp}}\right)$. The observed niche overlap (N-I) was compared with the null hypothesis $\left(\mathrm{N}_{\text {Samp }}-\mathrm{I}_{\text {Samp }}\right)$. Resampling was done using the number of occurrences recorded for the species in the given region, 120 times $(n=120)$. This number was chosen because it exceeded the general recommendation of $n=100$ by Broennimann et al..$^{25}$ and simplified hypothesis testing by allowing us to easily evaluate at $\alpha=0.050$ for two-tailed tests, where the null hypothesis was rejected if the observed value was more extreme than the top or bottom three null-model repetitions. For niche stability $(S)$ and niche overlap $(D)$, SAMP tests were conducted as one-tailed tests, which were considered significant if $S$ or $D$ was less than $95 \%$ of the null models. For other metrics, SAMP tests were two-tailed.

The SIM test ${ }^{25,27}$ asked whether a species' native and invasive ranges had more niche overlap or greater niche stability when compared with one another than when compared with other species. The null hypothesis was generated by measuring niche overlap between the observed bias-corrected introduced niche (I) and the native-range niches of all other species in the dataset $\left(\mathrm{N}_{\mathrm{Other}}\right)$, and between the observed bias-corrected native niche $(\mathrm{N})$ and the introduced-range niches of all other species $\left(\mathrm{I}_{\mathrm{Other}}\right)$. The null hypotheses $\left(\mathrm{N}_{\mathrm{Other}}-\mathrm{I}\right.$ and $\left.\mathrm{N}-\mathrm{I}_{\mathrm{Other}}\right)$ were then compared with the observed niche overlap (N-I). Because we computed niche overlap for every possible combination of species, the number of repetitions for the SIM test varied among comparisons, ranging from $n=91$ to $n=979$. This is a variation on the niche similarity test suggested by Warren et al. ${ }^{27}$ made possible by having a large number of species available for direct comparison. SIM tests were only performed on $S$ and $D$ and were always conducted as one-tailed tests.

The EQ test asked whether overlap between the bias-corrected native and introduced models was less than a pooled native/introduced model (N-I versus $\mathrm{NI}_{\text {Pooled }}-\mathrm{NI}_{\text {Pooled }}$ ). Thus, it asked whether the native and introduced models could be treated as equivalent. A significant EQ test meant that the native- and introducedrange models produced distinct predictions. For the EQ test, we resampled the actual native-range model $(\mathrm{N})$, actual invasive-range model (I) and pooled models $\left(\mathrm{NI}_{\text {Pooled }}\right) 100$ times. For the resampled $\mathrm{N}$ and I models, the number of occurrences in the resampled models equalled the number of occurrences in the species' native and introduced ranges. For the pooled models, one pooled model was resampled with the number of occurrences from the native range and one was resampled with the number of occurrences from the introduced range. The niche overlap of these two pooled models was then estimated. This method generated two sets of numbers: the niche overlap of 100 resampled N-I models and the niche overlap of 100 resampled $\mathrm{NI}_{\text {Pooled }}$ models. These two sets were compared using one-tailed $t$-tests, which were considered significant if the niche overlap of the N-I models was less than that of the pooled models. Initially, we tried comparing raw $\mathrm{N}$ and I models to the resampled $\mathrm{NI}_{\text {Pooled }}$ models (which had to be resampled), but during testing we found that failing to resample all models created small amounts of bias, particularly where occurrence densities were low. We were able to avoid this bias by also resampling the $\mathrm{N}$ and I models.

In summary, the SAMP test answered the question, 'Was the observed niche dynamic due to sampling artefacts or methodological biases?' The SIM test answered the question, 'Were the native and introduced niches more similar to one another than to the niches of other species?' and the EQ test answered the question, 'Were the native and introduced-range models the same?'

Source data. The species database comprised an initial set of 1,135 species native to every continent except Antarctica, with the median species being native to two continents and introduced to four continents. Occurrence data were downloaded from the GBIF (http://www.gbif.org) between January 2013 and June 2015. In June 2015, we appended records for all species whose coverage had improved since the first download date. Data in the United States were supplemented with occurrences from the Early Detection and Distribution Mapping System (http://www.eddmaps. org). We chose data sources that are widely used-maximizing the potential relevance of this study - and because they were amenable to our large-scale data collection effort. However, it is important to note that GBIF and Early Detection and Distribution Mapping System data have known biases ${ }^{28}$, although we have done our best to account for these, as described below. Overall, these datasets are very high resolution, with most occurrences having a precision much finer than the climate data. We cannot know the exact precision of each point in the database, but most, if not all, points were precise below the kilometre scale, often to the metre scale (Supplementary Fig. 1). In comparison, the climate maps formed a roughly $4 \mathrm{~km}^{2}$ grid.

We used information from the Catalogue of Life (http://www.catalogueoflife. org), European Network on Invasive Alien Species (http://www.nobanis.org), International Union for Conservation of Nature Red List (http://www.iucnredlist. org), Online Atlas of the British and Irish Flora (http://www.brc.ac.uk/plantatlas), US Department of Agriculture (USDA) Germplasm Resources Information Network (GRIN) Taxonomy Database (http://www.ars-grin.gov) and PLANTS 

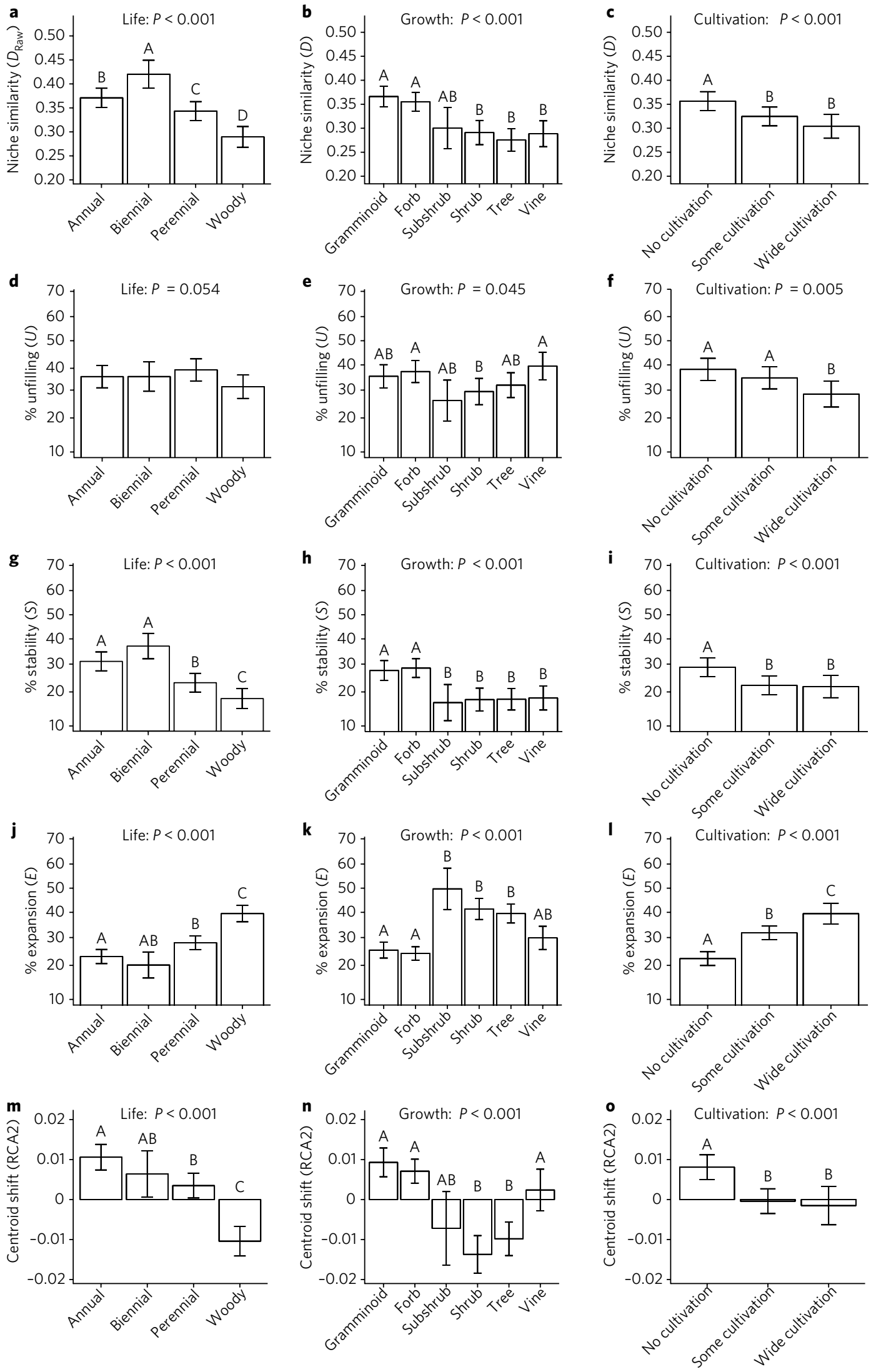

Fig. 5 | Effect of species traits on niche dynamics. a-I, Least-squared means \pm s.e. $(n=2,364)$ from models of niche overlap $\left(D_{\text {Raw: }}\right.$ : a-c); the angulartransformed percentage of analogue climate volume experiencing unfilling (U: $\mathbf{d}-\mathbf{f})$, stability (S: $\mathbf{g}-\mathbf{i})$ and expansion (E: $\mathbf{j}-\mathbf{I})$; and centroid shifts along RCA axis 2 (precipitation; $\mathbf{m}-\mathbf{0}$ ) against life span, growth form and cultivation. Significance is estimated using linear mixed models with Kenward-Rogers approximation (see Table 4). Post-hoc tests with Tukey correction are shown when factor effects are significant. Letters indicate the results of post-hoc Tukey comparisons, with different letters representing statistically significant differences among groups $(P \leq 0.050)$. 
Database (http://plants.usda.gov), as well as the primary and secondary literature to reconstruct the native and introduced ranges for each species. Occurrences identified as archaeophytes in the United Kingdom and Scandinavia were labelled as introduced. Each species distribution was visualized up to seven times to ensure that the occurrences and native or introduced assignments were correct. The USDA GRIN Database was used as a taxonomic authority and to determine each species' growth form and life cycle. We applied a 'lumping' approach, combining subspecies and any species with uncertain taxonomy, and duplicates were pared. These procedures resulted in 13.8 million records for 1,135 terrestrial plant species. As a result of excluding aquatic and parasitic species, those with low sample sizes and those with significant ranges in the Indopacific (for specific modelling concerns; see below), our final dataset included 815 species. All analyses were performed in $\mathrm{R}$ (ref. ${ }^{36}$ )

We accessed 2.5 arc-minute BIOCLIM current ( 1950-2000) global climate data from the WorldClim Global Climate Database (http://www.worldclim. org $/)^{37}$, a window that spans some, but not all, of the occurrence records. We did not adjust climatic conditions associated with individual occurrences to possible conditions at the time the occurrence was recorded. The 19 original BIOCLIM climate variables were reduced to four, using principal components analysis (Supplementary Table 3$)^{25}$, which encompassed $90 \%$ of the variance of the original variables. We used a varimax rotation to produce component scores that mapped clearly to changes in real climate variables using the 'psych' package ${ }^{38}$. The rotated components corresponded roughly to high temperature with low seasonality (RC1), high precipitation (RC2), high temperature with high seasonality (RC3) and low precipitation seasonality (RC4). The first two rotated components explained $60 \%$ of the total variance of all rotated components and were used to construct a $100 \times 100$ cell gridded climate space ${ }^{25}$. It was necessary to select only the top two components for ordination because niche unfilling, expansion and stability can only be evaluated in regions of climate space that are perfectly analogue in all dimensions. When three or more components were considered, a vast majority of the climate space was not analogue for all components, making direct niche comparisons irrelevant.

Niche modelling technique. Ordination was used to model climatic niche shifts because of its accuracy, robustness and efficiency ${ }^{25}$. Ordination is also appropriate for the descriptive, rather than predictive, modelling used in this study. This approach uses presence data to estimate the relative probability of a species occurring in any given region of climate space.

As described above, environmental niche models are susceptible to sampling biases produced by uneven representation of certain climates or overrepresentation of particular geographic areas ${ }^{39}$. For example, Western Europe is exhaustively sampled, whereas much of Central Asia is not, causing overrepresentation of Western European climates in uncorrected ordinations. This partly reflects the real distributions of the species and partly reflects geographic variation in the sampling effort $^{28}$. Rather than assuming that sampling was geographically uniform, we used the collective distribution of all species in the study as a proxy for sampling effort ${ }^{39}$ This technique removed geographic sampling effort bias and climate sampling bias and produced more conservative estimates of niche overlap (Supplementary Fig. 3) Caveats to this approach ${ }^{39,40}$ include the potential for inconsistent sampling across species, or for a slight part-whole correlation between each species distribution and the sampling effort estimate due to that species being one of over 1,100 species used to generate the sampling effort estimate. Estimating a unique sampling effort distribution for this large number of species was not feasible; however, the size of our database itself mitigates this part-whole correlation-even common species made up only a small fraction of the total number of records, resulting in negligible part-whole-correlation for most, if not all, species used in this study. The nullhypothesis tests were also designed so as to further account for bias caused by the part-whole correlation.

Sampling bias is removed from ordination analyses by dividing a probability distribution that describes the sampling bias $f_{\text {bis }}$ from the species' raw, uncorrected probability distribution $f_{\text {raw }}$. This can be done either before or after both probability distributions are smoothed. For smoothing, we used thin-plate spline regression with the 'fields' package ${ }^{41}$ in $\mathrm{R}$ ( ref. $^{36}$ ) and back-transformed. In our initial explorations, we found that spline-based smoothing outperformed Gaussian smoothing, especially for species with low sample sizes (data not shown). To avoid over- or under-smoothing, we restricted lambda to between 0.05 and 0.0005 .

We evaluated three methods of removing sampling bias. Each of these methods accomplishes the basic goal of dividing $f_{\text {raw }}$ by $f_{\text {bias }}$, the key difference being the step at which the distribution is smoothed:

Method 1: $f_{\text {niche }}=1+\operatorname{smooth}\left(f_{\text {raw }}-f_{\text {bias }}\right) / f_{\text {bias }}$

Method 2: $f_{\text {niche }}=\operatorname{smooth}\left(f_{\text {raw }}\right) / \operatorname{smooth}\left(f_{\text {bias }}\right)$

Method 3: $f_{\text {niche }}=\operatorname{smooth}\left(f_{\text {raw }} / f_{\text {bias }}\right)$

For each of these methods, $f_{\text {bias }}$ could be either: (1) an estimate of climate sampling bias, calculated as the frequency distribution of each set of climate variables on a given continent or (2) an estimate of both geographic and climate sampling bias, calculated as the probability of sampling each set of climate variables by randomly selecting an occurrence record from that continent. We compared the performance of these methods using MAXENT-estimated native- and introduced-range climate models for Cytisus scoparius and Sonchus asper used in a different study (D. Z. Atwater, unpublished data). The known starting models were randomly resampled 100 times in geographic space, projected into climate space and transformed using the above three methods, with bias correction using both the climate-only and geographic-plus-climate sources of sampling bias. We correlated the resampled models with the known models $(r)$ and compared the degree of native- and introduced-range niche overlap $\left(D_{\text {Raw }}\right)$ between the resampled models with the known model. To explore how the sample size affected model performance, we varied the number of occurrences, $n$, from 10-1,000 (the dataset had a median number of occurrences per region $n=207$ ). We also conducted EQ tests on the resampled models. These measurements identified method 3 as the best-performing of the three, having the highest $r$ and $D_{\text {Raw }}$ (Supplementary Fig. 4). This conclusion was further supported by visual analysis (Supplementary Fig. 5). To the eye, method 3 produced resampled models that most closely resembled the known models. It also produced the flattest null probability distribution (which should be equal at all points)

The reason method 3 worked best was that it produced reasonable estimates where values of $f_{\text {bias }}$ were very small (for example, at the edges of the climate distribution). In the other methods, small deviations in $f_{\text {bias }}$ and $f_{\text {raw }}$ amplified dramatically during division, especially at the edge of the climate distribution where $f_{\text {bias }}$ and $f_{\text {raw }}$ both approached zero. As a result, points along the edges of climate space $f_{\text {niche }}$ took extremely large values that constituted the majority of the probability mass distribution (evident as red dots in Supplementary Fig. 5, method 2 ). Our analysis also highlighted the importance of removing both geographic and climate sampling bias, as failing to exclude geographic sampling bias produced resampled models with poorer correlations with the known model, inappropriately high measurements of niche overlap (due to correlations among biased models) and clear visual evidence of bias (Supplementary Figs. 2 and 4).

Thus, for our final models, we removed sampling bias by dividing the species probability distribution by the geographic and climatic sampling bias, then smoothing using thin-plate splines. These bias-corrected estimates were scaled such that they integrated to one. This approach was functionally equivalent to other studies ${ }^{8,12,13,15,16}$, but was unique in accounting for geographic sampling bias in addition to climate sampling bias. Raster data were handled using the packages 'rgdal' ${ }^{42}$, 'raster' ${ }^{\text {'3 }}$ and 'dismo' ${ }^{44}$ in $\mathrm{R}$ (ref. ${ }^{36}$ ).

Niche comparisons. We used the revised biogeographic regions of Cox et al. ${ }^{26}$ to make biogeographic comparisons. Each occurrence was assigned to one of six regions: Africa (Arabia, the Sahara and Sub-Saharan Africa), Australasia (Australia, New Zealand and outlying islands), Eurasia (including Northern Africa Asia Minor, North and Central China, and the Himalayan Plateau), Indopacific (India, Southern China, Indochina and Maritime Southeast Asia), North America (excluding Southern Mexico) and South America (including Central America, Southern Mexico and the Caribbean). Comparisons involving the Indopacific were excluded because of low sample sizes, which confounded niche estimation and resulted in variable null hypotheses. Species with native occurrences in the Indopacific were also excluded from all GN-I models. For each species with at least 15 records on a continent, we separately estimated its climatic niche in each continent. Native and introduced distributions were modelled separately. We also modelled the global native range of each species by averaging $f_{\text {niche }}$ for each native range, weighted according to the relative representation of that species in the given range. We did this to reduce the influence of heavily sampled regions (for example, Eurasia) on the global estimate. Analyses were restricted to analogue climate space (climate space that is present in both ranges being compared) except where noted.

For each possible pair of native-native $(\mathrm{N}-\mathrm{N})$ and native-introduced $(\mathrm{N}-\mathrm{I}$ and GN-I) comparisons, we estimated niche overlap using Schoener's $D\left(\right.$ ref. ${ }^{29}$ ), which varies from zero (no overlap) to one (complete overlap). This metric is robust and accurate $^{30}$. Niche overlap was measured in raw climate space $\left(D_{\text {Raw }}\right)$ and climate space weighted according to the abundance of each climate in the introduced range $\left(D_{\mathrm{Clim}}\right)$ and the overall geographic sampling bias in the introduced range $\left(D_{\mathrm{Geo}}\right)$. These different metrics allowed us to assess niche overlap in raw climatic space $\left(D_{\text {Raw }}\right)$, the climates most common in the introduced range $\left(D_{\text {Clim }}\right)$ and the climates most heavily sampled $\left(D_{\mathrm{Geo}}\right)$.

We calculated $D$ for each regional comparison and evaluated differences in $D$ among species that varied in life span (annual, biennial, perennial or woody), growth form (gramminoid, forb, shrub, subshrub, tree or vine) and cultivation status (never, occasionally or widely; as per the USDA GRIN Taxonomy Database), using mixed models with continent (for example, native to Africa and introduced to Australia) and species random effects. For example, if a species was introduced to Africa and Eurasia and native to North America and South America, we performed four N-I comparisons (Africa to North America, Africa to South America, Eurasia to North America and Eurasia to South America), two GN-I comparisons (Africa to pooled native range and Eurasia to pooled native range) and one N-N comparison (North America to South America). Life span, growth form and cultivation status were never included together due to collinearity (Supplementary Table 2). The package 'lme4" ${ }^{35}$ was used to run these mixed models. The $F$ statistics were evaluated using Kenward-Rogers approximation, which is suitable for unbalanced incompletely nested designs ${ }^{46,47}$, using the packages 'ImerTest' ${ }^{48}$ and 'pbkrtest' ${ }^{47}$. We note that performing every N-I and GN-I combination amplified the degrees of freedom used for linear models, 
although the use of species and continent as random effects mitigated problems with error non-independence.

We estimated niche dynamics (for example, unfilling or expansion) by calculating the area of niche space in which a given proportion $(95 \%)$ of the occurrences were predicted to occur according to the corrected niche model $f_{\text {niche. }}$. We then measured the volume of the climatic niche that fell only in the native range (unfilling, $U$ ), only in the introduced range (expansion, $E$ ) and in both (stability, $S$ ), weighted according to the commonness of the climate in the introduced range (for expansion and stability) or native range (for unfilling) ${ }^{16}$. In non-analogue space, we measured the weighted volume of the climatic niche that was occupied in the native range but unavailable in the introduced range (abandonment, $A$ ) and the niche volume that was occupied in the introduced range but unavailable in the native range (pioneering, $P$ ). Abandonment was weighted according to native-range climate commonness and pioneering was weighted according to introduced-range climate commonness. To facilitate comparison with the null hypotheses, we expressed $A$ and $P$ as percentages by calculating the proportional contribution of $A$ and $P$ to total niche dynamics. For $U, S$ and $E$, we calculated the proportional contribution of each to the total analogue niche volume (by dividing by $U+S+E$ ).

We estimated directionality in climate shifts by measuring the centroid of each species probability mass function $f_{\text {niche }}{ }^{3}$ using the 'SDMTools' package ${ }^{49}$.

The probability function was square-root transformed before centroid estimation to minimize the effect of extreme values. A centroid shift along each climate axis (RC1 and RC2) was measured as the introduced-range centroid position minus the native-range position in Euclidean space. Centroid shifts were compared with cross-continent differences in available-climate centroids using linear models by subtracting the mean centroid shifts observed in the SAMP null models from the observed centroid shifts and then regressing those values against shifts in the centroid of available-climate space in the native and introduced range. Species and continental comparison were used as random effects.

Life Sciences Reporting Summary. Further information on experimental design is available in the Life Sciences Reporting Summary.

Data availability. The computer code and species database used in this study are publically accessible on VTechData, Virginia Tech's research data repository platform, at https://doi.org/10.7294/W41834NQ.

Received: 22 September 2016; Accepted: 27 October 2017; Published online: 04 December 2017

\section{References}

1. Silvertown, J. Plant coexistence and the niche. Trends Ecol. Evol. 19, 605-611 (2004)

2. Colwell, R. K. \& Rangel, T. F. Hutchinson's duality: the once and future niche. Proc. Natl Acad. Sci. USA 106, 19651-19658 (2009).

3. Guisan, A., Petitpierre, B., Broennimann, O., Daehler, C. \& Kueffer, C. Unifying niche shift studies: insights from biological invasions. Trends Ecol. Evol. 29, 260-269 (2014)

4. Pearman, P. B., Guisan, A., Broennimann, O. \& Randin, C. F. Niche dynamics in space and time. Trends Ecol. Evol. 23, 149-158 (2008).

5. Koop, A. L., Fowler, L., Newton, L. P. \& Caton, B. P. Development and validation of a weed screening tool for the United States. Biol. Invasions 14, 273-294 (2012).

6. Andersen, M. C., Adams, H., Hope, B. \& Powell, M. Risk assessment for invasive species. Risk Anal. 24, 787-793 (2004).

7. Veloz, S. D. et al. No-analog climates and shifting realized niches during the late quaternary: implications for 21st-century predictions by species distribution models. Glob. Change Biol. 18, 1698-1713 (2012).

8. Broennimann, O. et al. Evidence of climatic niche shift during biological invasion. Ecol. Lett. 10, 701-709 (2007).

9. Rodder, D. \& Lotters, S. Niche shift versus niche conservatism? Climatic characteristics of the native and invasive ranges of the Mediterranean house gecko (Hemidactylus turcicus). Glob. Ecol. Biogeogr. 18, 674-687 (2009).

10. Fitzpatrick, M. C., Weltzin, J. F., Sanders, N. J. \& Dunn, R. R. The biogeography of prediction error: why does the introduced range of the fire ant over-predict its native range? Glob. Ecol. Biogeogr. 16, 24-33 (2007).

11. Medley, K. A. Niche shifts during the global invasion of the Asian tiger mosquito, Aedes albopictus Skuse (Culicidae), revealed by reciprocal distribution models. Glob. Ecol. Biogeogr. 19, 122-133 (2010).

12. Gallagher, R. V., Beaumont, L. J., Hughes, L. \& Leishman, M. R. Evidence for climatic niche and biome shifts between native and novel ranges in plant species introduced to Australia. J. Ecol. 98, 790-799 (2010).

13. Early, R. \& Sax, D. F. Climatic niche shifts between species' native and naturalized ranges raise concern for ecological forecasts during invasions and climate change. Glob. Ecol. Biogeogr. 23, 1356-1365 (2014).
14. Liu, X. et al. Realized climatic niches are conserved along maximum temperatures among herpetofaunal invaders. J. Biogeogr. 44, 111-121 (2017)

15. Strubbe, D., Broennimann, O., Chiron, F. \& Matthysen, E. Niche conservatism in non-native birds in Europe: niche unfilling rather than niche expansion. Glob. Ecol. Biogeogr. 22, 962-970 (2013).

16. Petitpierre, B. et al. Climatic niche shifts are rare among terrestrial plant invaders. Science 335, 1344-1348 (2012).

17. Pimentel, D. et al. Economic and environmental threats of alien plant, animal, and microbe invasions. Agric. Ecosyst. Environ. 84, 1-20 (2001).

18. Perrings, C. et al. Biological invasion risks and the public good: an economic perspective. Ecol. Soc. 6, 1 (2002).

19. Wiens, J. J. et al. Niche conservatism as an emerging principle in ecology and conservation biology. Ecol. Lett. 13, 1310-1324 (2010).

20. Keane, R. M. \& Crawley, M. J. Exotic plant invasions and the enemy release hypothesis. Trends Ecol. Evol. 17, 164-170 (2002).

21. Shea, K. \& Chesson, P. Community ecology theory as a framework for biological invasions. Trends Ecol. Evol. 17, 170-176 (2002).

22. Webber, B. L., Le Maitre, D. C. \& Kriticos, D. J. Comment on "Climatic niche terrestrial plant invaders". Science 338, 193 (2012).

23. Li, Y., Liu, X., Li, X., Petitpierre, B. \& Guisan, A. Residence time, expansion toward the Equator in the invaded range and native range size matter to climatic niche shifts in non-native species. Glob. Ecol. Biogeogr. 23, 1094-1104 (2014).

24. Elith, J. \& Leathwick, J. R. Species distribution models: ecological explanation and prediction across space and time. Annu. Rev. Ecol. Syst. 40, 677-697 (2009).

25. Broennimann, O. et al. Measuring ecological niche overlap from occurrence and spatial environmental data. Glob. Ecol. Biogeogr. 21, 481-497 (2012).

26. Cox, C. B., Cottage, F. \& Close, B. The biogeographic regions reconsidered. J. Biogeogr. 28, 511-523 (2001).

27. Warren, D. L., Glor, R. E. \& Turelli, M. Environmental niche equivalency versus conservatism: quantitative approaches to niche evolution. Evolution 62, 2868-2883 (2008).

28. Meyer, C., Weigelt, P., Kreft, H. \& Lambers, J. H. R. Multidimensional biases, gaps and uncertainties in global plant occurrence information. Ecol. Lett. 19, 992-1006 (2016).

29. Schoener, T. The Anolis lizards of Bimini: resource partitioning in a complex fauna. Ecology 49, 704-726 (1968).

30. Rödder, D. \& Engler, J. O. Quantitative metrics of overlaps in Grinnellian niches: advances and possible drawbacks. Glob. Ecol. Biogeogr. 20, 915-927 (2011).

31. Jiménez-Valverde, A. et al. Use of niche models in invasive species risk assessments. Biol. Invasions 13, 2785-2797 (2011).

32. Václavík, T. \& Meentemeyer, R. K. Equilibrium or not? Modelling potential distribution of invasive species in different stages of invasion. Divers. Distrib. 18, 73-83 (2012).

33. Lee, C. E. Evolutionary genetics of invasive species. Trends Ecol. Evol. 17, 386-391 (2002).

34. Peterson, A. T. Ecological niche conservatism: a time-structured review of evidence. J. Biogeogr. 38, 817-827 (2011).

35. Guisan, A. \& Thuiller, W. Predicting species distribution: offering more than simple habitat models. Ecol. Lett. 8, 993-1009 (2005).

36. R Development Core Team R: A Language and Environment for Statistical Computing (R Foundation for Statistical Computing, 2017).

37. Hijmans, R. J., Cameron, S. E., Parra, J. L., Jones, P. G. \& Jarvis, A. Very high resolution interpolated climate surfaces for global land areas. Int. J. Climatol. 25, 1965-1978 (2005)

38. Revelle, A. W. \& Revelle, M. W. psych: Procedures for Psychological, Psychometic, and Rersonality Research R Package Version 1.5.4 (Northwestern University, Evanston, 2016).

39. Phillips, S. J. et al. Sample selection bias and presence-only distribution models: implications for background and pseudo-absence data. Ecol. Appl. 19, 181-197 (2009).

40. Yackulic, C. B. et al. Presence-only modelling using MAXENT: when can we trust the inferences? Methods Ecol. Evol. 4, 236-243 (2013).

41. Nychka, D., Furrer, R., Paige, J. \& Sain, S. fields: Tools for Spatial Data R Package Version 8.2-1 (University Corporation for Atmospheric Research, Boulder, 2015)

42. Bivand, R. et al. rgdal: Bindings for the 'Geospatial' Data Abstraction Library R Package Version 0.9-2 (Geospatial Data Abstraction Laboratory, 2016).

43. Hijmans, R. raster: Geographic Data Analysis and Modeling R Package Version 2.3-40 (2016).

44. Hijmans, R. J., Phillips, S., Leathwick, J. \& Elith, J. dismo: Species Distribution Modeling R Package Version 1.0-12 (2016).

45. Bates, D. M., Maechler, M., Bolker, B. \& Walker, S. Fitting linear mixedeffects models usinglme 4. J. Stat. Softw. 67, 1-48 (2015). 
46. Kenward, M. G. \& Roger, J. H. Small sample inference for fixed effects from restricted maximuml likelihood. Biometrics 53, 983-997 (1997).

47. Halekoh, U. \& Hojsgaard, S. A Kenward-Roger approximation and parametric bootstrap methods for tests in linear mixed models: the R package pbkrtest. J. Stat. Softw. 59, 1-30 (2014).

48. Kuznetsova, A., Brockhoff, P. B. \& Christensen, R. H. B. lmerTest: Tests in Linear Mixed Effects Models R Package Version 2.0-29 (R Foundation for Statistical Computing, Vienna, 2016).

49. VanDerWal, J., Falconi, L., Januchowski, S., Shoo, L. \& Storlie, C. SDMTools: Species Distribution Modelling Tools: Tools for Processing Data Associated with Species Distribution Modelling Exercises R Package Version $1.1-221$ (2014).

\section{Acknowledgements}

This work was partially supported by the Virginia Tech College of Agriculture and Life Sciences and the USDA's Controlling Weedy and Invasive Plants programme (201367013-21306).

\section{Author contributions}

J.N.B. and D.Z.A. conceived the study, which was refined by all authors. C.E. developed the species geographic databases and D.Z.A. refined them. D.Z.A. developed and performed all analyses with contributions from J.N.B. All authors discussed the results and contributed to writing the paper.

\section{Competing interests}

The authors declare no competing financial interests.

\section{Additional information}

Supplementary information is available for this paper at https://doi.org/10.1038/ s41559-017-0396-z.

Reprints and permissions information is available at www.nature.com/reprints. Correspondence and requests for materials should be addressed to D.Z.A.

Publisher's note: Springer Nature remains neutral with regard to jurisdictional claims in published maps and institutional affiliations. 


\section{natureresearch}

\section{Life Sciences Reporting Summary}

Nature Research wishes to improve the reproducibility of the work that we publish. This form is intended for publication with all accepted life science papers and provides structure for consistency and transparency in reporting. Every life science submission will use this form; some list items might not apply to an individual manuscript, but all fields must be completed for clarity.

For further information on the points included in this form, see Reporting Life Sciences Research. For further information on Nature Research policies, including our data availability policy, see Authors \& Referees and the Editorial Policy Checklist.

\section{- Experimental design}

\section{Sample size}

Describe how sample size was determined.

2. Data exclusions

Describe any data exclusions.

\section{Replication}

Describe whether the experimental findings were reliably reproduced.

4. Randomization

Describe how samples/organisms/participants were allocated into experimental groups.

5. Blinding

Describe whether the investigators were blinded to group allocation during data collection and/or analysis.
Sample size for most analyses (120) followed recommendations by Broennimann et al. Glob. Ecol. Biogeogr. 21:481- to exceed 100. In SIM tests the sample size was limited by the num. of available species for comparison $(n=91-979)$.

Data from the Indopacific were excluded because low occurrence density made it difficult to fit models. This is explained clearly in the text.

Experimental manipulation was not possible in this study.

Experimental manipulation was not used, but random resampling was used to generate null hypotheses.

Data were publicly available so blinding was not neccessary during data collection. Models were refined using only two species, so that we would be blinded to the results for the other 813 species.

Note: all studies involving animals and/or human research participants must disclose whether blinding and randomization were used.

\section{Statistical parameters}

For all figures and tables that use statistical methods, confirm that the following items are present in relevant figure legends (or in the Methods section if additional space is needed).

n/a $\mid$ Confirmed

$\bigotimes$ The exact sample size $(n)$ for each experimental group/condition, given as a discrete number and unit of measurement (animals, litters, cultures, etc.)

A description of how samples were collected, noting whether measurements were taken from distinct samples or whether the same

sample was measured repeatedly

$\bigotimes$ A statement indicating how many times each experiment was replicated

The statistical test(s) used and whether they are one- or two-sided (note: only common tests should be described solely by name; more

$\bigotimes$ complex techniques should be described in the Methods section)

$\square$ \ A description of any assumptions or corrections, such as an adjustment for multiple comparisons

$\square$ \ The test results (e.g. $P$ values) given as exact values whenever possible and with confidence intervals noted

$\square$ \ A clear description of statistics including central tendency (e.g. median, mean) and variation (e.g. standard deviation, interquartile range) $\square$ \ Clearly defined error bars 
Policy information about availability of computer code

\section{Software}

Describe the software used to analyze the data in this study.

For manuscripts utilizing custom algorithms or software that are central to the paper but not yet described in the published literature, software must be made available to editors and reviewers upon request. We strongly encourage code deposition in a community repository (e.g. GitHub). Nature Methods guidance for providing algorithms and software for publication provides further information on this topic.

\section{- Materials and reagents}

Policy information about availability of materials

\section{Materials availability}

Indicate whether there are restrictions on availability of unique materials or if these materials are only available for distribution by a for-profit company.

\section{Antibodies}

Describe the antibodies used and how they were validated for use in the system under study (i.e. assay and species).

10. Eukaryotic cell lines

a. State the source of each eukaryotic cell line used.

b. Describe the method of cell line authentication used.

c. Report whether the cell lines were tested for mycoplasma contamination.

d. If any of the cell lines used are listed in the database of commonly misidentified cell lines maintained by ICLAC, provide a scientific rationale for their use.
No unique materials were used.

No antibodies were used.

No eukaryotic cell lines were used.

No eukaryotic cell lines were used.

No eukaryotic cell lines were used.

No cell lines were used.

\section{- Animals and human research participants}

Policy information about studies involving animals; when reporting animal research, follow the ARRIVE guidelines

\section{Description of research animals}

Provide details on animals and/or animal-derived materials used in the study.
No animals were used.

Policy information about studies involving human research participants

\section{Description of human research participants}

Describe the covariate-relevant population characteristics of the human research participants.
This study did not involve human research participants. 